\title{
ON THE MULTIPLICITIES OF PLURI-ANTI-CANONICAL DIVISORS AND THE DEGREES OF FANO MANIFOLDS *
}

\author{
JUN-MUK HWANG HW $^{-}$ \\ Dedicated to Professor Yum-Tong Siu on his sixtieth birthday
}

\begin{abstract}
For a Fano manifold $X$ of Picard number 1, we study the relation between a bound on the multiplicities of effective pluri-anti-canonical divisors at a general point of $X$ and a bound on the degree of $X$. There is an obvious inequality relation between them which comes from asymptotic Riemann-Roch and a standard dimension-counting of Taylor polynomials. Refining the dimensioncounting by considering the behavior of multiplicities along standard rational curves, we show that there is a better inequality. This gives an improved bound on the degrees of Fano manifolds of Picard number 1.
\end{abstract}

1. Introduction. This work studies the relation between the following two problems.

Problem 1. Find a positive real number $A_{n}$ such that for any $n$-dimensional Fano manifold $X$ of Picard number $1,\left(-K_{X}\right)^{n} \leq A_{n}$.

Problem 2. Find a positive real number $B_{n}$ such that for any n-dimensional Fano manifold $X$ of Picard number 1 , a general point $x \in X$ and any $D \in\left|-k K_{X}\right|$ for sufficiently large $k$, the multiplicity $m_{x}(D)$ of $D$ at $x$ is bounded by $B_{n} k$.

Once an answer $B_{n}$ to Problem 2 is obtained, $A_{n}=\left(B_{n}\right)^{n}$ gives an answer to Problem 1, by asymptotic Riemann-Roch and standard dimension-counting of Taylor polynomials of the divisors at $x$. This was the approach taken in most works on Problem 1, e.g., [C1], [C2], $[\mathrm{KMM}],[\mathrm{N}],[\mathrm{R}]$ and $[\mathrm{RC}]$. That this is not the best approach to Problem 1 can be seen by the optimal answers expected:

Conjecture 1. For an n-dimensional Fano manifold $X$ of Picard number 1, $\left(-K_{X}\right)^{n} \leq(n+1)^{n}$.

Conjecture 2. For an n-dimensional Fano manifold $X$ of Picard number 1, a general point $x \in X$ and any $D \in\left|-k K_{X}\right|$ for sufficiently large $k, m_{x}(D) \leq 2 n k$.

Both conjectures are sharp. The bound in Conjecture 1 is realized by projective spaces and the bound in Conjecture 2 is realized by hyperquadrics. Conjecture 2 was proved in $[\mathrm{R}]$ and $[\mathrm{RC}]$ under the additional assumption that the tangent bundle $T(X)$ is semi-stable. For general $X$, the bound $A_{n}=\left(B_{n}\right)^{n}=n^{n}(n+1)^{n}$ was obtained in [C1], $[\mathrm{KMM}]$ and [N]. Their arguments can be easily improved to $A_{n}=\left(B_{n}\right)^{n}=$ $\left(\frac{n^{2}+4 n+3}{4}\right)^{n}([\mathrm{C} 2])$.

The discrepancy between optimal values of $A_{n}$ and $\left(B_{n}\right)^{n}$ suggests that their should be an argument finer than the standard dimension-counting from asymptotic Riemann-Roch. In [H2], Conjecture 1 was proved for $n=4$. The key ingredient is a refined dimension-counting of Taylor polynomials, which was done by considering the geometry of the vectors tangent to standard rational curves (see the definition

\footnotetext{
${ }^{*}$ Received March 19, 2003; accepted for publication July 22, 2003.

†Korea Institute for Advanced Study, 207-43 Cheongnyangni-dong, Seoul, 130-722, Korea (jmhwang@ns.kias.re.kr). Supported by the Korea Research Foundation Grant (KRF-2002-070C00003).
} 
below). This refined counting was possible because one could use sharp RiemannRoch for $h^{0}\left(X, \mathcal{O}\left(-K_{X}\right)\right)$ in dimension 4 . I gave a talk on this result in a conference at Princeton in 2002. During the talk, Yum-Tong Siu asked whether the arguments could be generalized to give an improved answer to Problem 1 also in higher dimensions. I answered negatively based on the following reasoning. In dimension $\geq 5$, it is unlikely that we can use sharp Riemann-Roch in this problem. So we have to use asymptotic Riemann-Roch. The improvement coming from the geometry of vectors tangent to standard rational curves, as given in [H2], is of lower order, so would not affect the leading term of asymptotic Riemann-Roch. Thus the method would not give improvement if $n \geq 5$. After the talk, however, Lawrence Ein suggested to me that if one takes into account the higher vanishing orders along the variety of vectors tangent to standard rational curves, the argument might work even for asymptotic Riemann-Roch. This suggestion was the starting point of the current work. Our main result is the following. Recall that a standard rational curve in $X$ is an immersion $h: \mathbf{P}_{1} \rightarrow X$ with $h^{*} T(X) \cong \mathcal{O}(2) \oplus \mathcal{O}(1)^{d-2} \oplus \mathcal{O}^{n-d+1}$ where $d$ is the degree of the curve with respect to $-K_{X}, 2 \leq d \leq n+1$. Any Fano manifold has standard rational curves.

Theorem 1. Let $X$ be an n-dimensional Fano manifold of Picard number 1. Suppose $X$ contains a standard rational curve of degree $d$. Assume that there exists a real number $\gamma>d$ with the following property: for a general point $x \in X$ and any $D \in\left|-k K_{X}\right|$ with $k>>0$, its multiplicity $m_{x}(D)$ at $x$ is $\leq \gamma k$. Then

$$
\left(-K_{X}\right)^{n} \leq \gamma^{n}+2\left(\gamma-\frac{3}{2} d\right)^{n}-3(\gamma-d)^{n}
$$

Suppose, furthermore, that $d \geq 3$. Then

$$
\left(-K_{X}\right)^{n} \leq \gamma^{n}-(\gamma-d)^{n-1}(\gamma+(n-1) d) .
$$

In $[\mathrm{C} 2,5.6]$, it was proved that $\gamma=(n+2-d) d$ satisfies the condition of Theorem 1. Thus we get the following corollary, giving an improvement of the known bounds for $A_{n}$.

COROLlary 1. Let $X$ be an n-dimensional Fano manifold of Picard number 1 of dimension $n$. Assume that $X$ contains a standard rational curve of degree $d=2$. Then

$$
\begin{aligned}
\left(-K_{X}\right)^{n} & \leq(2 n)^{n}+2(2 n-3)^{n}-3(2 n-2)^{n} \\
& \approx(0.345 \ldots)(2 n)^{n} \quad \text { for large } n .
\end{aligned}
$$

If $X$ contains a standard rational curve of degree $d \geq 3$. Then

$$
\left(-K_{X}\right)^{n} \leq(n+2-d)^{n} d^{n}-(n+1-d)^{n-1}(2 n+1-d) d^{n} .
$$

When $T(X)$ is semi-stable, we can use $\gamma=2 n$ from $[\mathrm{R}]$ and $[\mathrm{RC}]$ to get the following.

Corollary 2. Let $X$ be an n-dimensional Fano manifold of Picard number 1. Assume that $X$ contains a standard rational curve of degree $d \geq 3$ and $T(X)$ is 
semi-stable. Then

$$
\begin{aligned}
\left(-K_{X}\right)^{n} & \leq(2 n)^{n}-(2 n-d)^{n-1}(2 n+d(n-1)) \\
& \leq(2 n)^{n}-(n-1)^{n-1}\left(n^{2}+2 n-1\right) .
\end{aligned}
$$

When $n=5$ or $6, T(X)$ is semi-stable by [H1]. Moreover, we may assume that $X$ contains a standard rational curve of degree $2 \leq d \leq n$ by [CMS]. Thus we get the following bound in dimensions 5 and 6 from Corollary 1 and Corollary 2 .

Corollary 3. For a 5-dimensional Fano manifold $X$ of Picard number 1, $\left(-K_{X}\right)^{5} \leq 81250$. For a 6-dimensional Fano manifold $X$ of Picard number 1 , $\left(-K_{X}\right)^{6} \leq 2659392$.

Although these bounds are much weaker than the conjectured bounds $6^{5}=7776$ and $7^{6}=117649$, no better bounds seem to be known.

In Section 2, the key idea of refined dimension-counting of Taylor polynomials is explained. Section 3 is devoted to an upper bound for the polynomial interpolations, which will be used in Section 4 to prove Theorem 1 .

Acknowledgment. I am very grateful to Lawrence Ein for the suggestion to look at the higher order vanishing along the variety of standard rational tangents, which was the starting point of this work. I would like to thank Youngook Choi for an interesting discussion on polynomial interpolations.

2. Refined dimension-counting of Taylor polynomials. Let $X$ be an $n$ dimensional Fano manifold of Picard number 1. A standard rational curve of degree $d$ in $X$ is an immersion $h: \mathbf{P}_{1} \rightarrow X$ with $h^{*} T(X) \cong \mathcal{O}(2) \oplus \mathcal{O}(1)^{d-2} \oplus \mathcal{O}^{n+1-d}$. It is well-known that standard rational curves of some degree $d$ satisfying $2 \leq d \leq n+1$ sweep out an open subset of $X$. For a general point $x \in X$, let $\mathcal{C}_{x} \subset \mathbf{P} T_{x}(X)$ be the closure of the union of vectors tangent to a standard rational curve through $x$ of a fixed degree $d$. Each component of $\mathcal{C}_{x}$ has dimension $d-2$ by the basic deformation theory of rational curves. Let $\mathcal{J}_{x}$ be the homogeneous ideal defining the subvariety $\mathcal{C}_{x} \subset \mathbf{P} T_{x}(X)$ and $\mathcal{J}_{x}^{<k>}$ be its $k$-th symbolic power, namely,

$$
\left.\mathcal{J}_{x}^{<k>}:=\text { polynomials vanishing on } \mathcal{C}_{x} \text { to order } \geq k\right\} .
$$

For an irreducible subvariety $Y \subset X$ and an effective divisor $D$, let $m_{Y}(D)$ be the multiplicity of $D$ at a general point of $Y$. Then we have the following result.

Proposition 1. Denote $\mathbf{P} T_{x}(X)$ by E. For $r \geq k d$, the codimension of the set

$$
\left\{D \in\left|-k K_{X}\right|, m_{x}(D) \geq r+1\right\}
$$

in $\left|-k K_{X}\right|$ is at most

$$
\begin{gathered}
h^{0}(E, \mathcal{O})+h^{0}(E, \mathcal{O}(1))+\cdots+h^{0}(E, \mathcal{O}(k d-1))+h^{0}(E, \mathcal{O}(k d))+ \\
h^{0}\left(E, \mathcal{J}_{x}(k d+1)\right)+h^{0}\left(E, \mathcal{J}_{x}^{<2>}(k d+2)\right)+\cdots+h^{0}\left(E, \mathcal{J}_{x}^{<r-1-k d>}(r-1)\right)+ \\
h^{0}\left(E, \mathcal{J}_{x}^{<r-k d>}(r)\right) .
\end{gathered}
$$


Proof. Let $C \subset X$ be a standard rational curve of degree $d$ passing through a general point $x \in X$. Recall Nadel's product lemma ([N], also see [H2, Proposition 9] for a simple proof):

Lemma 1. For $D \in\left|-k K_{X}\right|, m_{C}(D) \geq m_{x}(D)-k d$.

Set $q:=m_{x}(D)$ and assume that $q>k d$ so that $C \subset D$. Set $\ell:=m_{C}(D)$. Let $\varphi: \tilde{X} \rightarrow X$ be the blow-up of $X$ at $x$. Then $\varphi^{*} D=q E+\tilde{D}$ where $\tilde{D}$ is the proper transform of $D$ and $E$ is the exceptional divisor, $E \cong \mathbf{P} T_{x}(X)$. The restriction $D^{\prime}:=\tilde{D} \mid E$ is the tangent cone of $D$ at $x$. Let $\tilde{C}$ be the proper transform of $C$. Then

$$
\ell=m_{\tilde{C}}(\tilde{D})
$$

implies that

$$
m_{z}(\tilde{D}) \geq \ell
$$

for $z=\tilde{C} \cap E$. It follows that

$$
m_{z}\left(D^{\prime}\right) \geq \ell \geq q-k d
$$

for any $z \in \mathcal{C}_{x}$. Thus if $q=m_{x}(D) \geq k d$, then

$$
D^{\prime} \in \mathbf{P} H^{0}\left(E, \mathcal{J}_{x}^{<q-k d>}(q)\right) .
$$

This proves Proposition 1.

The trouble with Proposition 1 is that the number $h^{0}\left(E, \mathcal{J}_{x}^{<i>}(k d+i)\right)$ is very hard to compute. To start with, we know very little about the geometry of $\mathcal{C}_{x}$ for general $X$. However even in the case where $\mathcal{C}_{x} \subset \mathbf{P} T_{x}(X)$ is a well-known projective variety, the computation of $h^{0}\left(\mathbf{P} T_{x}(X), \mathcal{J}_{x}^{<i>}(k d+i)\right)$ for large $i$ is very difficult. Of course, to use Proposition 1, it suffices to have a good upper bound of $h^{0}\left(\mathbf{P} T_{x}(X), \mathcal{J}_{x}^{<i>}(k d+i)\right)$. An easy upper bound is given by $h^{0}\left(\mathbf{P} T_{x}(X), \mathbf{m}_{\alpha}^{i}(k d+i)\right)$ where $\mathbf{m}_{\alpha}$ is the ideal for a point $\alpha \in \mathcal{C}_{x}$. We can do better by considering finitely many points on $\mathcal{C}_{x}$. This is is exactly what we will do in this paper.

3. An upper bound on the dimension of polynomial interpolations. Let $x_{1}, \ldots, x_{m}$ be $m \geq 2$ distinct points on $\mathbf{P}_{n}$. Let $\mathcal{I}$ be the homogeneous ideal defining the reduced scheme of their union and $\mathcal{I}^{<k>}$ be its $k$-th symbolic power. In this section, we will get an upper bound of $h^{0}\left(\mathbf{P}_{n}, \mathcal{I}^{<q-c>}(q)\right)$ where $c$ and $q$ are positive integers with $c<q$. This belongs to the classical problem of polynomial interpolations (cf. [Ci], [M] for surveys). The upper bound we will consider is rather elementary. Since we cannot find a good reference where the bound below is stated, we will give full details.

Proposition 2. Let $\nu(n, q):=h^{0}\left(\mathbf{P}_{n}, \mathcal{I}^{<q-c>}(q)\right)$ where $\mathcal{I}$ is as above. Assume that $c \geq n(m-1)$ and $c$ is a multiple of $m-1$.

(i) If $q \leq \frac{m c}{m-1}$,

$$
\nu(n, q) \leq\left(\begin{array}{c}
n+q \\
n
\end{array}\right)-m\left(\begin{array}{c}
n+q-c-1 \\
n
\end{array}\right)
$$

(ii) If $q \geq \frac{m c}{m-1}+1$,

$$
\nu(n, q) \leq\left(\begin{array}{c}
n+q \\
n
\end{array}\right)+(m-1)\left(\begin{array}{c}
q-\frac{m c}{m-1}+n-1 \\
n
\end{array}\right)-m\left(\begin{array}{c}
n+q-c-1 \\
n
\end{array}\right)
$$


Proof. To prove the proposition, we may assume that $x_{1}, \ldots, x_{m}$ are collinear. The reason is as follows. Let $\mathbf{P}_{n}=\mathbf{P} V$ for a vector space $V$ of dimension $n+1$. Choose a basis $v_{0}, \ldots, v_{n}$ of $V$ such that, when $x_{i}=\left[a_{i}^{0} v_{0}+\cdots+a_{i}^{n} v_{n}\right]$ for all $i, 1 \leq i \leq m$,

$(\dagger)$ the numbers $a_{i}^{0}$ and $a_{i}^{1}$ are non-zero for all $i$, and $\left\{\frac{a_{i}^{0}}{a_{i}^{1}}, 1 \leq i \leq m\right\}$ are distinct $m$ complex numbers.

Consider the linear transformations $\left\{\phi_{\lambda} \in G L(V), \lambda \in \mathbf{C}^{*}\right\}$ defined by

$$
\phi_{\lambda}\left(v_{0}\right)=v_{0}, \quad \phi_{\lambda}\left(v_{1}\right)=v_{1}, \quad \phi_{\lambda}\left(v_{i}\right)=\lambda v_{i} \text { for } 2 \leq i \leq n .
$$

Then

$$
h^{0}\left(\mathbf{P}_{n}, \mathcal{I}^{<q-c>}(q)\right)=h^{0}\left(\mathbf{P}_{n}, \phi_{\lambda}^{*} \mathcal{I}^{<q-c>}(q)\right)
$$

for all $\lambda \in \mathbf{C}^{*}$. On the other hand, as $\lambda$ approaches 0 , the points $\left\{\phi_{\lambda}\left(x_{1}\right), \ldots, \phi_{\lambda}\left(x_{m}\right)\right\}$ converges to $m$ distinct collinear points by the assumption $(\dagger)$. Thus by upper-semicontinuity, an upper bound of $\nu(n, q)$ for the collinear case gives an upper bound for the general case.

From now on, we will assume that $x_{1}, \ldots, x_{m}$ are collinear. Then it is easy to see that the symbolic power agrees with the ordinary power, i.e., $\mathcal{I}^{<k>}=\mathcal{I}^{k}$ for all $k$. For example, this follows from the result of [Ho] and the fact that the union of collinear points is a complete intersection.

Recall that a finite subscheme of length $d$ is $d$-regular (e.g., [La, I.1.8.30]). Thus

$$
H^{1}\left(\mathbf{P}_{n}, \mathcal{I}^{q-c}(q)\right)=0 \quad \text { if } \quad q \leq \frac{m c}{m-1} .
$$

So (i) follows from the exact sequence

$$
0 \longrightarrow H^{0}\left(\mathbf{P}_{n}, \mathcal{I}^{q-c}(q)\right) \longrightarrow H^{0}\left(\mathbf{P}_{n}, \mathcal{O}(q)\right) \longrightarrow \sum_{i=1}^{m} H^{0}\left(x_{i}, \mathcal{O} / \mathbf{m}_{i}^{q-c}\right) \longrightarrow 0
$$

where $\mathbf{m}_{i}$ denotes the maximal ideal of the point $x_{i}$.

To prove (ii), let $\mathbf{P}_{n-1} \subset \mathbf{P}_{n}$ be a hyperplane containing $x_{1}, \ldots, x_{m}$. As long as $q-1-c \geq 0$, we have an exact sequence

$$
0 \longrightarrow H^{0}\left(\mathbf{P}_{n}, \mathcal{I}^{q-1-c}(q-1)\right) \longrightarrow H^{0}\left(\mathbf{P}_{n}, \mathcal{I}^{q-c}(q)\right) \longrightarrow H^{0}\left(\mathbf{P}_{n-1}, \mathcal{I}^{q-c}(q)\right),
$$

which gives

$$
\text { (甘) } \nu(n, q) \leq \nu(n-1, q)+\nu(n, q-1) \text { if } q-c \geq 1 \text {. }
$$

For convenience, define $\nu(n, q):=0$ if $n$ is a non-positive integer and $q \geq c$. Since $\nu(1, q)=h^{0}\left(\mathbf{P}_{1}, \mathcal{O}(q-m(q-c))\right)= \begin{cases}0 & \text { if } m c+(1-m) q \leq-1 \\ m c+(1-m) q+1 & \text { if } m c+(1-m) q \geq 0\end{cases}$ we can see that the inequality $(\sharp)$ holds for all integers $n$ as long as $q \geq c$. 
Using $(\sharp)$ and Proposition $2(\mathrm{i})$, we can calculate $\nu(n, q)$ for any $q \geq \frac{m c}{m-1}+1$ as follows. Repeatedly applying $(\sharp)$, we get

$$
\begin{aligned}
\nu(n, q) & \leq \nu(n-1, q)+\nu(n, q-1) \\
& \leq \nu(n-2, q)+2 \nu(n-1, q-1)+\nu(n, q-2) \\
& \leq \nu(n-3, q)+3 \nu(n-2, q-1)+3 \nu(n-1, q-2)+\nu(n, q-3) \\
& \leq \cdots \\
& \leq \sum_{i=0}^{M}\left(\begin{array}{c}
M \\
i
\end{array}\right) \nu(n-i, q-M+i)
\end{aligned}
$$

where $M$ is any positive integer satisfying $q-M \geq c+1$. Let us choose $M=$ $q-\frac{m c}{m-1}+n-1 \geq 1$. Then

$$
q-M=\frac{m c}{m-1}-n+1=c+\frac{c}{m-1}-n+1 \geq c+1
$$

by the assumption $c \geq n(m-1)$. Since $\nu(n-i, q-M+i)=0$ for $i \geq n$, we have

$$
\nu(n, q) \leq \sum_{i=0}^{n-1}\left(\begin{array}{c}
M \\
i
\end{array}\right) \nu(n-i, q-M+i)
$$

For $i \leq n-1$, we have $q-M+i \leq q-M+n-1=\frac{m c}{m-1}$ and, by Proposition 2 (i),

$$
\begin{aligned}
\nu(n-i, q-M+i) & =\left(\begin{array}{c}
(n-i)+(q-M+i) \\
n-i
\end{array}\right)-m\left(\begin{array}{c}
(n-i)+(q-M+i)-c-1 \\
n-1
\end{array}\right) \\
& =\left(\begin{array}{c}
n+q-M \\
n-i
\end{array}\right)-m\left(\begin{array}{c}
n+q-M-c-1 \\
i
\end{array}\right) .
\end{aligned}
$$

Using the identity

$$
\left(\begin{array}{c}
A \\
0
\end{array}\right)\left(\begin{array}{c}
B \\
p
\end{array}\right)+\left(\begin{array}{c}
A \\
1
\end{array}\right)\left(\begin{array}{c}
B \\
p-1
\end{array}\right)+\cdots+\left(\begin{array}{c}
A \\
p
\end{array}\right)\left(\begin{array}{c}
B \\
0
\end{array}\right)=\left(\begin{array}{c}
A+B \\
p
\end{array}\right)
$$

we have

$$
\begin{aligned}
\sum_{i=0}^{n-1}\left(\begin{array}{c}
M \\
i
\end{array}\right)\left(\begin{array}{c}
n+q-M \\
n-i
\end{array}\right) & =\left(\begin{array}{c}
n+q \\
n
\end{array}\right)-\left(\begin{array}{c}
M \\
n
\end{array}\right) \\
\sum_{i=0}^{n-1}\left(\begin{array}{c}
M \\
i
\end{array}\right)\left(\begin{array}{c}
n+q-M-c-1 \\
n-i
\end{array}\right) & =\left(\begin{array}{c}
n+q-c-1 \\
n
\end{array}\right)-\left(\begin{array}{c}
M \\
n
\end{array}\right) .
\end{aligned}
$$

Thus we conclude

$$
\nu(n, q) \leq\left(\begin{array}{c}
n+q \\
n
\end{array}\right)+(m-1)\left(\begin{array}{c}
M \\
n
\end{array}\right)-m\left(\begin{array}{c}
n+q-c-1 \\
n
\end{array}\right)
$$

for $q \geq \frac{m c}{n-1}+1$, which proves Proposition (ii). 
4. Refined dimension-counting arising from finitely many standard rational curves. Let us go back to the situation of Section 2. Select $m$ distinct points $x_{1}, \ldots, x_{m}$ from $\mathcal{C}_{x}$. Let $\mathcal{I}$ be the ideal defining their union. Then

$$
h^{0}\left(E, \mathcal{J}_{x}^{<q-k d>}(q)\right) \leq h^{0}\left(E, \mathcal{I}^{<q-k d>}(q)\right)=\nu(n-1, q)
$$

for $q>k d$, where we set $c=d k$ in the definition of $\nu(n, q)$. Let us assume that $k$ is a multiple of $(m-1)$ and $k \geq(m-1) n$ so that $c=d k$ is also a multiple of $(m-1)$ and $c \geq(m-1) n$. Then we can apply Proposition 2 to $\nu(n-1, q)$.

Proposition 3. Let $m \geq 2$ be an integer, $k$ be a multiple of $(m-1)$ with $k \geq(m-1)(n-1)$ and $d$ be an integer, $d \geq 2$. Let $x_{1}, \ldots, x_{m}$ be distinct points on $\mathbf{P}_{n-1}$ and define $\nu(n-1, q)=h^{0}\left(\mathbf{P}_{n-1}, \mathcal{I}^{<q-d k>}(q)\right)$ for positive integers $q>d k$. Then for any integer $r>\frac{m d k}{m-1}$, we have

$$
\begin{aligned}
& \sum_{j=1}^{r-d k} \nu(n-1, d k+j) \leq \\
& \left(\begin{array}{c}
n+r \\
n
\end{array}\right)-\left(\begin{array}{c}
n+d k \\
n
\end{array}\right)-m\left(\begin{array}{c}
n+r-d k-1 \\
0
\end{array}\right)+(m-1)\left(\begin{array}{c}
n+r-\frac{m d k}{m-1}-1 \\
n
\end{array}\right) .
\end{aligned}
$$

Proof. Since $c=d k$ satisfies the assumption of Proposition 2,

$$
\begin{aligned}
& \sum_{j=1}^{\frac{m d k}{m-1}-d k} \nu(n-1, d k+j)= \\
& \sum_{j=1}^{\frac{m d k}{m-1}-d k}\left[\left(\begin{array}{c}
n-1+d k+j \\
n-1
\end{array}\right)-m\left(\begin{array}{c}
n-1+d k+j-d k-1 \\
0
\end{array}\right)\right]
\end{aligned}
$$

and

$$
\sum_{j=\frac{m d k}{m-1}-d k+1}^{r-d k} \nu(n-1, d k+j)=\sum_{i=1}^{r-\frac{m d k}{m-1}} \nu\left(n-1, \frac{m d k}{m-1}+i\right)
$$

is bounded by

$$
\begin{gathered}
\sum_{j=\frac{m d k}{m-1}-d k+1}^{r-d k}\left(\begin{array}{c}
n-1+\frac{m d k}{m-1}+i \\
n-1
\end{array}\right)+(m-1) \sum_{i=1}^{r-\frac{m d k}{m-1}}\left(\begin{array}{c}
\frac{m d k}{m-1}+i-\frac{m d k}{m-1}+n-2 \\
n-1
\end{array}\right) \\
-m \sum_{j=\frac{m d k}{m-1}-d k+1}^{r-d k}\left(\begin{array}{c}
n-1+\frac{m d k}{m-1}+i-d k-1 \\
n-1
\end{array}\right) .
\end{gathered}
$$


Thus $\sum_{j=1}^{r-d k} \nu(n-1, d k+j)$ is bounded by

$$
\begin{aligned}
& \sum_{j=1}^{r-d k}\left[\left(\begin{array}{c}
n-1+d k+j \\
n-1
\end{array}\right)-m\left(\begin{array}{c}
n-1+j-1 \\
n-1
\end{array}\right)\right] \\
& +(m-1) \sum_{i=1}^{r-\frac{m d k}{m-1}}\left(\begin{array}{c}
n-1+i-1 \\
n-1
\end{array}\right) \\
& =\left(\begin{array}{c}
n+r \\
n
\end{array}\right)-\left(\begin{array}{c}
n+d k \\
n
\end{array}\right)-m\left(\begin{array}{c}
n+r-d k-1 \\
0
\end{array}\right)+(m-1)\left(\begin{array}{c}
n+r-\frac{m d k}{m-1}-1 \\
n
\end{array}\right),
\end{aligned}
$$

which proves the proposition.

Proposition 4. Suppose there exist $m$ standard rational curves of degree $d$ through a general point $x \in X$ which are smooth at $x$ and give $m$ distinct tangent vectors there. For any positive integer $k$ divisible by $m-1$ and $d k \geq n(m-1)$, if $r>d k$ is a positive integer satisfying

$$
\begin{aligned}
& \left(\begin{array}{c}
n+r \\
n
\end{array}\right)-m\left(\begin{array}{c}
n+r-d k-1 \\
0
\end{array}\right)+(m-1)\left(\begin{array}{c}
n+r-\frac{m d k}{m-1}-1 \\
n
\end{array}\right) \\
& <h^{0}\left(X, \mathcal{O}\left(-k K_{X}\right)\right),
\end{aligned}
$$

then there exists $D \in\left|-k K_{X}\right|$ with $m_{x}(D)>r$.

Proof. By Proposition 1, it suffices to show that the number

$$
\begin{gathered}
h^{0}(E, \mathcal{O})+h^{0}(E, \mathcal{O}(1))+\cdots+h^{0}(E, \mathcal{O}(k d-1))+h^{0}(E, \mathcal{O}(k d))+ \\
h^{0}\left(E, \mathcal{J}_{x}(k d+1)\right)+h^{0}\left(E, \mathcal{J}_{x}^{<2>}(k d+2)\right)+\cdots+h^{0}\left(E, \mathcal{J}_{x}^{<r-1-k d>}(r-1)\right) \\
+h^{0}\left(E, \mathcal{J}_{x}^{<r-k d>}(r)\right)
\end{gathered}
$$

is bounded above by

$$
\left(\begin{array}{c}
n+r \\
n
\end{array}\right)-m\left(\begin{array}{c}
n+r-d k-1 \\
0
\end{array}\right)+(m-1)\left(\begin{array}{c}
n+r-\frac{m d k}{m-1}-1 \\
n
\end{array}\right)
$$

Since

$$
h^{0}(E, \mathcal{O})+h^{0}(E, \mathcal{O}(1))+\cdots+h^{0}(E, \mathcal{O}(k d-1))+h^{0}(E, \mathcal{O}(k d))=\left(\begin{array}{c}
n+d k \\
n
\end{array}\right)
$$

this follows from Proposition 3 and the fact that

$$
h^{0}\left(E, \mathcal{J}_{x}^{<q-k d>}(q)\right) \leq h^{0}\left(\mathbf{P}_{n-1}, \mathcal{I}^{<q-k d>}(q)\right)
$$

for any $q>k d$.

We are ready to prove Theorem 1.

Proof of Theorem 1. By Proposition 4, if there exists $\gamma>d$ such that any $D \in\left|-k K_{X}\right|$ with $k$ divisible by $m-1$ and $d k \geq n(m-1)$ satisfies $m_{x}(D) \leq \gamma k$, then

$$
\begin{aligned}
& \left(\begin{array}{c}
n+\gamma k \\
n
\end{array}\right)-m\left(\begin{array}{c}
n+\gamma k-d k-1 \\
0
\end{array}\right)+(m-1)\left(\begin{array}{c}
n+\gamma k-\frac{m d k}{m-1}-1 \\
n
\end{array}\right) \\
& \geq h^{0}\left(X, \mathcal{O}\left(-k K_{X}\right)\right) .
\end{aligned}
$$


Comparing the leading coefficients of both sides using asymptotic Riemann-Roch

$$
h^{0}\left(X, \mathcal{O}\left(-k K_{X}\right)\right)=\frac{\left(-K_{X}\right)^{n}}{n !} k^{n}+O\left(k^{n-1}\right),
$$

we see that

$$
\gamma^{n}+(m-1)\left(\gamma-\frac{m d}{m-1}\right)^{n}-m(\gamma-d)^{n} \geq\left(-K_{X}\right)^{n} .
$$

When $X$ is a Fano manifold of Picard number 1, there are at least three standard rational curves of equal degree through a general point having distinct tangent directions ([H2, Proposition 2]). Putting $m=3$, we get Theorem 1 . When $d \geq 3$, there are $(d-2)$-dimensional family of standard rational curves through $x$. Thus we may take $m \rightarrow \infty$. Since

$$
\lim _{m \rightarrow \infty}(m-1)\left[(\gamma-d)^{n}-\left(\gamma-d-\frac{d}{m-1}\right)^{n}\right]=n(\gamma-d)^{n-1} d
$$

and

$$
\left(-K_{X}\right)^{n} \leq \gamma^{n}-(\gamma-d)^{n}-(m-1)\left[(\gamma-d)^{n}-\left(\gamma-d-\frac{d}{m-1}\right)^{n}\right]
$$

we get

$$
\begin{aligned}
\left(-K_{X}\right)^{n} & \leq \gamma^{n}-(\gamma-d)^{n}-n(\gamma-d)^{n-1} d \\
& =\gamma^{n}-(\gamma-d)^{n-1}(\gamma+(n-1) d) .
\end{aligned}
$$

\section{REFERENCES}

[C1] CAmpana, F., Une version géométrique généralisée du théorème du produit de Nadel, Bull. Soc. Math. France, 119 (1991), pp. 479-493.

[C2] Campana, F., Un théorème de finitude pour les variétés de Fano suffisamment uniréglées, Géometrie Complexe (Paris, 1992), Actualites Sci. Indust., 1438 Hermann, Paris (1996), pp. 23-33.

[Ci] Ciliberto, C., Geometric aspects of polynomial interpolation in more variables and of Waring's problem, European Congress of Mathematics Vol. I (Barcelona, 2000), pp. 289-316, Progr. Math., 201, Birkhauser, Basel, 2001.

[CMS] Cho, K., MiYaoka, Y. and Shepherd-Barron, N. I., Characterizations of projective space and applications to complex symplectic manifolds, In Higher dimensional birational geometry (Kyoto, 1997), Adv. Stud. Pure Math., 35 (2002), pp. 1-88.

[Ho] Hochster, M., Criteria for equality of ordinary and symbolic powers of primes, Math. Z., 133 (1973), pp. 53-65.

[H1] Hwang, J.-M., Stability of tangent bundles of low dimensional Fano manifolds with Picard number 1, Math. Annalen, 312 (1998), pp. 599-606.

[H2] Hwang, J.-M., On the degrees of Fano four-folds of Picard number 1, J. reine angew. Math., 556 (2003), pp. 225-235.

[KMM] Kollár, J., MrYaOKa, Y. AND Mori, S., Rational curves on Fano varieties, In Proc. Alg. Geom. Conf. Trento, Lect. Notes in Math., 1515 (1992), pp. 100-105.

[L] Lazarsfeld, R., Positivity in algebraic geometry, to appear.

[N] NADEL, A., The boundedness of degree of Fano varieties with Picard number one, J. AMS, 4 (1991), pp. 681-692.

[M] Miranda, R., Linear systems of plane curves, Notices Amer. Math. Soc., 46 (1999), pp. 192-201.

[R] RAN, Z., On semipositivity of sheaves of differential operators and the degree of a unipolar Q-Fano variety, In Symposium in Honor of C. H. Clemens (Salt Lake City, UT, 2000), Contemp. Math., 312 (2002), pp. 177-186.

[RC] Ran, Z. And Clemens, H., A new method in Fano geometry, Intern. Math. Research Notices, 2000, pp. 527-549. 
